family therapy and effectively using the group for supervision.

It would also appear that this was a cost-effective use of a psychiatrist/psychotherapist's time, in that for one-and-a-half hours per week 30 health care staff were able to have supervision and the numbers of patients with psychological problems treated by the primary care team over the year increased. I have attempted to describe the process of increasing the effectiveness of the staff group in this paper and hope this may be useful for facilitators in other primary care teams.

\section{Acknowledgements}

This work was done under the auspices of the Tavistock Clinic. I wish to thank especially $\mathrm{Dr}$ Stanford Bourne for comments and discussion of this paper.

\section{Reference}

Bion, W. R. (1961) Experiences in Groups. London: Tavistock Publications, p. 129.

\title{
Improving psychiatric day hospital attendance - a Liverpool intervention study
}

\author{
G. T. Ridley, Registrar, Mersey Regional Psychiatry Training Scheme; K. Clare, \\ Charge Nurse; M. E. DEwEY, Lecturer in Psychological Statistics; and I. A. DAvidson, \\ Consultant Psychiatrist, University Department of Psychiatry, Royal Liverpool \\ Hospital, Liverpool L7 8XP
}

In spite of the somewhat disordered development of day care for the mentally ill, the Psychiatric Day Hospital (PDH) has become accepted as an essential element in the mental health services. Several recent studies have defined a role for the PDH in the management of more acute psychiatric disorders (Creed et al, 1989), and this is likely to become increasingly important with the continuing reduction in the availability of in-patient facilities.

An area which has received relatively little attention in the literature, but which is likely to become increasingly important with greater reliance on day hospital care, is that of a poor overall attendance rate. Few studies address this issue directly, but, defining non-attendance as failure to attend beyond the first day of admission, studies of consecutive PDH referrals report rates of roughly $50 \%$, while those including some form of post-referral screening report rates nearer to $20 \%$ (Dick, 1986). It therefore seems possible that poor attendance at PDH may be improved by a relatively simple intervention, and it is the purpose of this study to address this issue specifically.

\section{The study}

The Royal Liverpool Hospital PDH is a 40-place unit on the second floor of a large teaching hospital, adjacent to the university department of psychiatry and two wards, and serving an inner city area containing the most underprivileged wards in Liverpool.

Staff consists of a charge nurse, three staff nurses, a senior occupational therapist, an occupational therapy assistant, and a full-time registrar. Characteristics of the day patient population are similar to those nationally (Conway et al, 1988).

Non-attendance rate was estimated in 1988 to be roughly $50 \%$, and, in an effort to improve on this, an assessment package was introduced in early 1989 , comprising a semi-structured interview by a senior nurse, a visit to the unit and a booklet describing its activities. The effects of this were studied by examining case notes of consecutive referrals to the unit eight months pre-intervention and eight months post-intervention, for information on age, sex, DSM-III diagnosis, employment and marital status, attendance, and time between referral and admission. Figures were collected separately for those attending by ambulance and those travelling by other means.

No changes were made in the availability or range of specific treatments offered either within or outside the unit during the study period, and referring doctors were unaware that the intervention had been implemented. 


\section{Findings}

A total of 159 patients were studied, 91 in the non-ambulance group (57 pre-intervention and 34 post-intervention) and 68 in the ambulance group (46 and 22 respectively). The age range was $19-88$, with patients allocated to 10-year age bands for statistical purposes. DSM-III diagnoses were divided into six groups by chapter: phobic (agoraphobia, social phobia), anxiety (generalised anxiety disorder, obsessive compulsive disorder), affective (major depressive episode, hypomanic episode), organic (organic delusional syndrome, dementia organic affective syndrome), schizophrenic (schizophrenia, schizo-affective disorder, paranoid disorder) and other (anorexia nervosa, adjustment disorder, schizotypal personality disorder).

No differences were found in the clinicodemographic characteristics of the pre- and postintervention groups studied, and none of the individual variables studied was demonstrated to have any independent effect on attendance rates. Within the group not attending by hospital transport, significant differences were found between non-attendance rates pre- and post intervention $(P=0.0013-0.0074)$. Overall non-attendance rates in this group were $50 \%$ prior to the intervention and fell to $20 \%$ following its implementation (95\% confidence interval 1.32-12.09). Mean time between referral and admission was 24.5 days pre-intervention and 23.5 days post-intervention. Non-attendance rates in the ambulance group were unaffected by the intervention, being $28 \%$ and $27 \%$ respectively before and after its use.

\section{Comment}

This was a retrospective study of all consecutive referrals to a PDH within a specified time period; no attempt was made at matching the control and study groups for any individual variable, and it is recognised that this introduces a source of bias in the results. However, none of the variables studied differed significantly between pre- and postintervention patients and, in common with a recent study of general hospital out-patients (Frankel $e t$ al, 1989), no association was demonstrated between demographic characteristics and non-attendance. The intervention produced a fall in non-attendance rates of $30 \%$, the respective figures of $50 \%$ and $20 \%$ being similar to those quoted in previous studies of consecutive admissions and admissions following some form of post-referral screening. Clearly, post-referral interventions have produced a reduction in non-attendance rates in other populations, although the issue has not previously been addressed specifically.

Intervention trials in other settings have indicated that telephone and postal contact with referrals can significantly improve attendance rates, and it seems likely that the relevant common factor between these various interventions is one of improved communication. This may explain the improved attendance rate of patients offered hospital transport in this study.

No attempt has been made here to explore the reasons for poor attendance, though lack of adequate communication and poor understanding by patients of the reason for referral have been implicated by other workers (Frankel et al, 1989); the intervention used here is likely to have improved those factors considerably. A further factor implicated in other studies has been relatively short notice given to subsequent non-attenders; this study did not confirm this, with no difference in time between referral and admission being observed.

While the setting for this study has been a psychiatric day hospital, the variety of other specialities in which poor attendance has been improved by simple interventions, such as general medical and geriatric clinics, is suggestive that its findings may have wider applicability: any potential improvement in the currently rather poor situation from a simple, cheap, audit-based intervention is clearly worthy of further study on a prospective, case-control basis.

\section{References}

Conway, M., Davidson, I. A. \& Dewey, M. E. (1988) Who uses a psychiatric day hospital and for how long? A Liverpool study. Bulletin of the Royal College of Psychiatrists, 13, 62-65.

Creed, F., ANthony, P., Goodbert, K. et al (1989). Treatment of severe psychiatric illness in a day hospital. British Journal of Psychiatry, 154, 341-347.

Dicx, P. H. (1986) Letter. Day and full-time psychiatric treatment. British Journal of Psychiatry, 148, 477-478.

Frankel, S., Farrow, A. \& West, R. (1989) Nonattendance or non-invitation? A case-control study of failed outpatient appointments. British Medical Journal, ii, 1343-1345.

A complete list of references and detailed statistical analysis is available from Dr Ridley. 